\title{
Eficacia en campo de un repelente a base de para-mentano-3,8-diol y aceite de limonaria contra Culicoides pachymerus (Diptera: Ceratopogonidae) en Colombia
}

\author{
Erika Santamaría', Olga Lucía Cabrera'1, Yaneth Zipa², Raúl Hernando Pardo ${ }^{3}$ \\ 1 Grupo de Entomología, Instituto Nacional de Salud, Bogotá, D.C., Colombia \\ 2 Grupo de Enfermedades Transmitidas por Vectores, Secretaría de Salud de Boyacá, Tunja, Colombia \\ ${ }^{3}$ Grupo de Entomología y Enfermedades Transmitidas por Vectores, Universidad de La Salle, Bogotá, D.C., \\ Colombia \\ Introducción. Culicoides pachymerus, por sus picaduras, ocasiona una grave molestia sanitaria en la \\ población del occidente del departamento de Boyacá (Colombia). \\ Objetivo. Evaluar de forma preliminar en campo el efecto de una loción repelente con ingredientes \\ activos de origen natural ( $\mathrm{p}$-mentano-3,8-diol $16 \%$ y aceite de limonaria $2 \%$ ) contra las picaduras de \\ C. pachymerus. \\ Materiales y métodos. En parejas de voluntarios ubicadas en el peridomicilio, se comparó \\ simultáneamente la loción repelente con un control (sin aplicación de repelente) mediante la tasa de \\ picadura de $C$. pachymerus en el antebrazo, el porcentaje de protección y el tiempo de protección \\ durante un periodo entre tres y seis horas después de la aplicación. La prueba se repitió 10 veces. \\ Resultados. Solo dos hembras de $C$. pachymerus se recolectaron en el tratamiento con la loción \\ repelente, mientras que, en el control, la tasa promedio de picadura fue de 47,7 hembras por persona \\ en 10 minutos. El porcentaje de protección promedio del repelente fue de $100 \%$ hasta cuatro horas \\ después de la aplicación y se mantuvo alto $(99,5 \%)$ hasta cinco horas después. Solo en dos pruebas \\ se registró picadura confirmada de C. pachymerus, con un tiempo de protección de 332,2 y 338,2 \\ minutos. En las ocho pruebas restantes el tiempo de protección superó la duración de la prueba. \\ Conclusión. El repelente evaluado demostró una gran eficacia en la prevención de las picaduras de \\ C. pachymerus, hasta cinco horas después de su aplicación.
}

Palabras clave: Ceratopogonidae, repelentes de insectos, Colombia.

doi: http://dx.doi.org/10.7705/biomedica.v32i3.738

Field efficacy of repellent formulation containing para-menthane-3,8-diol and lemongrass against Culicoides pachymerus (Diptera: Ceratopogonidae) in Colombia

Introduction. Culicoides pachymerus is a major pest species for the inhabitants of the western Boyacá province of Colombia.

Objective. The effect of a repellent lotion based on p-menthane-3,8-diol (16\%) and lemongrass oil $(2 \%)$ was evaluated against the bites of $C$. pachymerus.

Materials and methods. The repellent lotion was compared simultaneously with a control (no treatment) by human landing catches of $C$. pachymerus on the forearms of paired volunteers situated near human dwellings. Protection percentage and protection time for 3 to $6 \mathrm{~h}$ after repellent application was calculated. The test was repeated ten times.

Results. Only two females of $C$. pachymerus were collected on arms with the repellent treatment. In contrast, the mean biting rate in the untreated control was 47.7 midges/person/10 min. Mean protection percentage of the repellent was $100 \%$ up to $4 \mathrm{~h}$ and $99.5 \%$ up to $5 \mathrm{~h}$. Protection time was 332.2 and $338.2 \mathrm{~min}$ in the two replicates where bites of $C$. pachymerus were confirmed. In the remaining eight replicates protection time exceeded the test duration.

Conclusion. The repellent showed high efficacy against $C$. pachymerus, up to $5 \mathrm{~h}$ post-application.

Key words: Ceratopogonidae, insect repellent, Colombia.

doi: http://dx.doi.org/10.7705/biomedica.v32i3.738 
En el área rural del piedemonte de la Cordillera Oriental que rodea el valle del río Magdalena en el departamento de Boyacá, Culicoides pachymerus Lutz,1914 es una molestia sanitaria seria que llega a causar problemas dermatológicos por sus altas tasas de picadura (promedios geométricos de hasta 51,8 picaduras por persona en cinco minutos) (1). Como una de las medidas de protección contra las picaduras, se ha recomendado el uso de repelentes; sin embargo, su elevado costo limita el acceso a gran parte de la comunidad.

El objetivo del estudio fue evaluar, de forma preliminar, la eficacia en campo del repelente de origen natural "NO MÁS" contra las picaduras de $C$. pachymerus. Este repelente ha sido eficaz contra vectores de malaria $(2,3)$ con gran aceptabilidad y bajo costo; para el año 2007, se estimó que la aplicación diaria por persona valía aproximadamente US\$ 0,025, en el Perú (información disponible en: www.delcielo.net/no-mas-repellent.php).

\section{Materiales y métodos}

El estudio se llevó a cabo en el área rural del municipio de San Pablo de Borbur (Boyacá), en las veredas Chizo Cuepar $\left(05^{\circ} 37^{\prime} \mathrm{N}\right.$ y $\left.74^{\circ} 04^{\prime} \mathrm{O}\right)$ y San Isidro $\left(05^{\circ} 39^{\prime} \mathrm{N}\right.$ y $\left.74^{\circ} 03^{\prime} \mathrm{O}\right)$, con altitudes entre los 450 y los $670 \mathrm{msnm}$. Para el municipio se reporta una temperatura media de $23,4{ }^{\circ} \mathrm{C}$ y una precipitación media anual de $2.620 \mathrm{~mm}$ (4).

De los métodos de ensayo disponibles para probar repelentes de mosquitos en campo (5), se empleó el de la Organización Mundial de la Salud debido a que es uno de los más empleados para cuantificar la protección y la duración del efecto de formulaciones repelentes (6).

Se registró la tasa de picadura de $C$. pachymerus sobre parejas de voluntarios ubicados en el peridomicilio de cinco viviendas. Los voluntarios de cada pareja recibieron uno de los siguientes tratamientos: (a) loción repelente "NO MÁS", con dos ingredientes activos: p-mentano-3,8-diol al $16 \%$, extraído de Corymbia citriodora, y aceite de limonaria al $2 \%$, extraído de las hojas de Cymbopogon citratus, aplicado a una dosis de 2 $\mathrm{ml} / 1.000 \mathrm{~cm}^{2}$ de superficie de piel y (b) control: ningún producto. La parte del cuerpo expuesta

Correspondencia:

Erika Santamaría, Grupo de Entomología, Instituto Nacional de Salud, Bogotá, D.C., Colombia.

Teléfono (571) 2207700 , extensión 1341

esantamaria@ins.gov.co

Recibido: 01/12/11; aceptado:07/05/12 a las picaduras fue el antebrazo izquierdo. Las demás partes del cuerpo estuvieron protegidas, incluyendo las manos (cubiertas con guantes de látex) y la cabeza (cubierta con una careta de muselina).

Tres horas antes de iniciar la prueba (15:00), los voluntarios se lavaron el antebrazo que iba a estar expuesto con un jabón sin fragancia y abundante agua fría, y se le aplicó el repelente al voluntario correspondiente. Luego, permanecieron sentados en un lugar cubierto con la instrucción de no tocar ni humedecer el antebrazo tratado hasta el momento de la prueba. La duración de cada prueba fue de tres horas, entre las 15:00 y las 18:00, periodo de mayor actividad de picadura de $C$. pachymerus en el área de estudio (7).

Mediante recolección con un pincel humedecido en alcohol, se registró el número de Culicoides que se acercó a picar a cada voluntario durante los 10 minutos iniciales de las 15:00, las 16:00 y las 17:00. Esta fracción de tiempo se definió para proteger a los voluntarios de un excesivo número de picaduras. Además, se registró la hora en la cual la primera y la segunda hembras lograron alimentarse (hembra con abdomen dilatado de color rojo). Cuando durante los primeros 10 minutos no se acercó a picar ningún culicoide, el registro del tiempo se extendió hasta cuando fue necesario, dentro del periodo de duración de la prueba.

Los tratamientos y la posición de los voluntarios en el peridomicilio, la cual se rotó cada hora, fueron asignados aleatoriamente. Para evitar una posible interferencia del tratamiento con repelente sobre el control, la distancia entre los voluntarios fue de, por lo menos, $20 \mathrm{~m}$.

Los ejemplares de Culicoides recolectados se preservaron en alcohol al $70 \%$ hasta su llegada al laboratorio, en donde fueron contados e identificados hasta especie teniendo en cuenta el patrón de pigmentación de las alas y las descripciones originales de varias especies $(8,9)$. La prueba se repitió dos veces en cada una de las cinco viviendas, una en mayo y la otra en noviembre de 2009, para un total de 10 repeticiones.

La eficacia del repelente se determinó mediante el cálculo de dos indicadores: el porcentaje de protección definido por la fórmula: $(A-B / A)^{\star} 100$, en donde $A$ es el número de insectos que pican en el control en determinado periodo y $\mathrm{B}$ es el número de insectos que pican al voluntario con el repelente en el mismo periodo (10), y el tiempo 
de protección, que son los minutos transcurridos desde la aplicación del producto hasta la primera picadura, tiempo confirmado por una segunda picadura en los 30 minutos siguientes $(10,11)$.

El método empleado en las pruebas de eficacia del repelente fue aprobado por el Comité de Ética en Investigación del Instituto Nacional de Salud.

\section{Resultados}

Durante las pruebas se capturaron 1.972 Culicoides, todas hembras, de las que el 99,5\% fueron identificadas como $C$. pachymerus; las otras especies recolectadas fueron $C$. paraensis (8 hembras) y $C$. debilipalpis (1 hembra).

Los resultados de las pruebas muestran una aparente gran eficacia de la loción repelente evaluada en la prevención de las picaduras de C. pachymerus (cuadro 1). Mientras que en el tratamiento con el repelente se recolectaron dos hembras de $C$. pachymerus, en el control, el promedio geométrico de la tasa de picadura fue de 47,7 hembras por persona en 10 minutos $\left(\mathrm{IC}_{95 \%}\right.$ : $24,8-90,6)$. La protección fue total hasta cuatro horas después de la aplicación y se mantuvo muy alta, con un porcentaje promedio de $99,5 \%$, a las cinco horas después de la aplicación. En cuanto al tiempo de protección, en ocho de las diez pruebas, los voluntarios que usaron el repelente fueron protegidos completamente de las picaduras de $C$. pachymerus (sin registro de picadura confirmada) hasta la finalización de la prueba (seis horas después de la aplicación). En las otras dos pruebas, el tiempo de protección fue de 332,2 y 338,2 minutos. Para efectos de comparación, en el control, la mediana del tiempo en el que se presentó la primera picadura confirmada fue de tan solo 2,0 minutos $\left(\mathrm{IC}_{95 \%}: 1,2-2,3\right)$.

\section{Discusión}

Aunque, en general, se considera que los repelentes a base de componentes de origen vegetal tienen una duración limitada (12), la formulación evaluada en este estudio presentó una buena duración de su efecto repelente, que se podría extender más allá del tiempo de observación (seis horas). Otras formulaciones repelentes a base de p-mentano3,8-diol han demostrado una protección de larga duración contra otras especies de ceratopogónidos que son molestia sanitaria. Por ejemplo, para $C$. impunctatus se registró una protección de $98 \%$ hasta ocho horas después de la aplicación (13), para C. variipennis, cinco horas de protección en pruebas de laboratorio (14), y para Leptonocops carteri, una duración del efecto repelente mayor de seis horas (11).

La excelente protección por un tiempo de cinco horas del repelente evaluado contra $C$. pachymerus, sumada al hecho de que esta especie presenta su mayor actividad solo durante tres horas del día (15:00 a 18:00), mientras que en el resto del día su actividad es muy reducida (7), sugieren que una sola aplicación diaria por persona del repelente hacia las 14:00 sería suficiente para reducir notablemente las picaduras de $C$. pachymerus en el área de estudio. Sin embargo, es necesario hacer pruebas adicionales en las que se evalúe el efecto de factores bióticos como el sudor humano, reconocido por reducir la acción de los repelentes. Además, se deben considerar factores abióticos como la temperatura, la humedad y la luz solar, los cuales pueden afectar la absorción, la evaporación y la estabilidad de la estructura química del repelente en la piel (5).

En conclusión, bajo las condiciones descritas, la loción repelente a base de p-mentano-3,8-diol y aceite de limonaria demostró una gran eficacia en la prevención de las picaduras de $C$. pachymerus, con una protección de 99,5\% o más hasta cinco horas después de su aplicación. Se recomienda, entonces, realizar estudios detallados que incluyan el efecto de la sudoración, la aceptabilidad por parte de la población y el seguimiento de los posibles

Cuadro 1. Protección de la loción repelente (p-mentano-3,8-diol y aceite de limonaria) contra las picaduras de C. pachymerus en relación con el tiempo posterior a la aplicación $(n=10)$

\begin{tabular}{cccc}
\hline & Número promedio de insectos ${ }^{1}\left(\mathbf{I C}_{95 \%}\right)$ & Repelente & $\begin{array}{c}\text { Porcentaje de } \\
\text { protección }\end{array}$ \\
\hline $\begin{array}{c}\text { Tiempo después de aplicar } \\
\text { el repelente (horas) }\end{array}$ & Control & 0 & 100 \\
\hline 3 & $46,9(24,9-87,8)$ & 0 & 100 \\
4 & $50,2(26,9-93,0)$ & $0,15(0-0,4)$ & 99,5 \\
\hline
\end{tabular}

${ }_{1}^{1}$ Promedio geométrico de Williams de hembras recolectadas los 10 primeros minutos de cada hora 
efectos secundarios que el uso del repelente pueda tener sobre la salud en la población afectada por C. pachymerus en el área de estudio.

\section{Agradecimientos}

A Sam Darling de la Fundación del Cielo, por el suministro de la loción repelente evaluada, y a Marco Fidel Suárez y Mauricio Carrillo, por su apoyo durante las pruebas.

\section{Conflicto de intereses}

Los autores declaran que no existe conflicto de intereses.

\section{Financiación}

Instituto Nacional de Salud, Universidad de La Salle, Secretaría de Salud de Boyacá y Colciencias (proyecto código: 2104-04-16499).

\section{Referencias}

1. Santamaría E, Cabrera OL, Zipa Y, Ahumada M, Ferro C, Pardo R. Diagnóstico preliminar de la molestia sanitaria causada por Culicoides (Diptera: Ceratopogonidae) en el departamento de Boyacá, Colombia. Biomédica. 2008;28:497-509.

2. Moore SJ, Darling ST, Sihuincha M, Padilla N, Devine GJ. A low-cost repellent for malaria vectors in the Americas: Results of two field trials in Guatemala and Peru. Malar J. 2007;6:101. http://dx.doi.org/10.1186/1475-2875-6-101

3. Kiszewski AE, Darling ST. Estimating a mosquito repellent's potential to reduce malaria in communities. $\mathrm{J}$ Vector Borne Dis. 2010;47:217-21.

4. Instituto Geográfico Agustín Codazzi. Diccionario geográfico de Colombia. Tercera edición. Santafé de Bogotá: Reprolaser Ltda.; 1996. p. 2076-7.
5. Barnard DR. Biological assays methods for mosquito repellents. J Am Mosquito Control Assoc. 2005;21(Suppl.):12-6.

6. World Health Organization. Guidelines for efficacy testing of mosquito repellents for human skin. Geneva: WHO; 2009. p. 1-30.

7. Carrasquilla MC. Patrones temporales y sitios de cría de Culicoides pachymerus (Diptera: Psychodidae) en el departamento de Boyacá, Colombia. (tesis). Bogotá: Universidad de los Andes; 2008.

8. Wirth WW, Dyce AL, Spinelli GR. An atlas of wing photographs, with a summary of the numerical character of the neotropical species of Culicoides (Diptera: Ceratopogonidae). Contrib Am Ent Inst. 1998;25:1-72.

9. Forattini OP. Culicoides da Regiao Neotropical (Diptera: Ceratopoginidae). Arq Fac Hig Saude Publica Univ Sao Paulo. 1957;11:161-526.

10. Schreck CE. Techniques for the evaluation of insect repellents: A critical review. Ann Rev Entomol. 1977;22:10119. http://dx.doi.org/10.1146/annurev.en.22.010177.000533

11. Carroll SP, Loye J. Field test of a lemon eucalyptus repellent against Leptoconops biting midges. J Am Mosq Control Assoc. 2006;22:483-5. http://dx.doi.org/10.2987/8756971X(2006)22[483:FTOALE]2.0.CO;2

12. Fradin MS, Day JF. Comparative efficacy of insect repellents against mosquito bites. N Engl J Med. 2002;347:13-8.

13. Trigg JK. Evaluation of a eucalyptus-based repellent against Culicoides impunctatus (Diptera: Ceratopogonidae) in Scotland. J Am Mosq Control Assoc. 1996;12:329-30.

14. Trigg JK, Hill N. Laboratory evaluation of a eucalyptusbased repellent against four biting arthropods. Phytother Res. 1996;10:313-6. http://dx.doi.org/10.1002/(SICl)10991573(199606)10:4<313::AID-PTR854>3.0.CO;2-O 